\title{
Entrelacs
}

Cinéma et audiovisuel

5 | 2005

La Machine

\section{Le monde allant Verne}

Frédéric Cassan

\section{OpenEdition}

\section{Journals}

Édition électronique

URL : http://journals.openedition.org/entrelacs/151

DOI : 10.4000/entrelacs. 151

ISSN : 2261-5482

Éditeur

Éditions Téraèdre

Édition imprimée

Date de publication : 1 novembre 2005

Pagination : 151-157

ISBN : 978-2-912868-70-1

ISSN : $1266-7188$

Référence électronique

Frédéric Cassan, «Le monde allant Verne », Entrelacs [En ligne], 5 | 2005, mis en ligne le 01 août 2012, consulté le 19 avril 2019. URL : http://journals.openedition.org/entrelacs/151 ; DOI : 10.4000/ entrelacs.151

Ce document a été généré automatiquement le 19 avril 2019

Tous droits réservés 


\title{
Le monde allant Verne
}

\author{
Frédéric Cassan
}

1 De l'incidence immédiate à l'influence plus structurelle, un regard sur la manière dont l'œuvre de Jules Verne s'immisce "machinalement» dans la création et le paysage audiovisuel. Transposer formellement les œuvres de Jules Verne au cinéma pose essentiellement et quasi-systématiquement la question de la conception visuelle. La plasticité unique de son travail peut être autant le conducteur assuré d'une riche expérience visuelle, une manne fantastique pour un cinéma de genre très inspiré, comme l'incommensurable défit du metteur en scène qui souhaite en faire l'adaptation officielle. Verne est le créateur d'univers fantastiques tellement paradoxaux, parfaitement identifiés dans notre patrimoine imaginaire, ce presque palpable scientifique où l'on place souvent son œuvre dans un très sérieux désordre épistémologique, et qui pourtant, dans la plus évidente poésie du bricolage, demeure d'une naïveté enfantine. Ce double langage, ce creuset d'inventions aussi fameuses que fumeuses, la source illustre des confusions et croyances qui entourent étrangement le caractère novateur de l'auteur est par ailleurs l'une des plus grandes difficultés d'adaptation et peut être, à la fois, son plus bel effet.

\section{Adopte : Une époque, un Musée (Jules Verne et ses transformations)}

2 Un voyage extraordinaire c'est bien plus qu'un paysage et son panorama. Il s'agit d'un acte, un choix d'idéal, renoncer à quelques balises connues et s'entourer d'une force enfantine sublimante : plonger avec Nemo.

Cette liberté qui détermine tout le génie créatif de l'auteur fait autant appel à la géographie qu'à la machinerie pour décrire les ailleurs. Le lieux comme la méthode pour s'y rendre sont les deux faces d'une même vision du voyage. Le comment et le pourquoi d'une exploration semblent ingrédients et vecteurs d'une rêverie savante et complexe, une alchimie fine et précieuse qui coopère du fantasme et de la relique, du mythe inaccessible à la fonction plus charnelle des objets, un dosage précis qui caractérise 
l'œuvre de Verne. Les machines sont toute aussi étranges et fascinantes que les mondes qu'elles visitent. L'univers de Verne est profondément mythologique mais très concrètement, en apothicaire des paradis et enfers perdus, la boutique du vieil homme (le grenier du jeune enfant) regorge toujours de fournitures expressives, denrées rares, collectes risquées, outils de rêve, instruments de cauchemar, les traces du destin (souvenirs): la bimbeloterie et l'atelier d'un voyageur onirique. L'époque de Verne est profondément encyclopédiste. C'est l'univers fourmillant de tous les herbiers venimeux, fossiles marins et papillons exotiques. Les collections diverses restent des entassements épars qu'il faut encore rassembler, l'exposition des Arts n'est pas à la tendance dépouillée et minimaliste. Le Muséum d'histoire naturelle est encore un véritable fourre-tout ou les «monstres" presque reconstitués du passé côtoient de vraies espèces vivantes et ou l'invraisemblance et le banal se juxtapose dans l'urgence à la limite de la confusion.

4 Au début du XIX ${ }^{e}$ siècle le général et diplomate écossais, Lord Elgin, profite d'un jeu d'alliance pour organiser le démontage et pillage partiel du Parthénon. Beaucoup de pièces sont détruites ou perdues, une partie très détériorée est finalement racheté par le gouvernement britannique pour rejoindre momies et sarcophage au British Museum. C'est l'époque d'une abondance amassée de la connaissance, d'un rapt idéaliste dans les affaires culturelles des peuples, nos miroirs "indigènes", et les vestiges d'un monde, certains l'entrevoient, qui ne durera pas. On découvre et classe à tour de bras les nouvelles espèces animales, on ne trace plus les contours de continents lointains mais on en explore toujours le cœur. L'Afrique est toujours le terrain d'expéditions aussi dangereuses qu'hallucinantes, animées d'une énergie aventurière volontaire et à la fois très dogmatique. Une époque encore assez folle ou l'on disserte en salon du savoir acquis et expérimente dans la jungle. En avant scène les artistes sont autant accumulateurs que leur monde est archiviste. Les ateliers regorgent d'un séduisant capharnaüm, des morceaux de mémoire éparses. Cette attitude est particulièrement contestataire puisqu'il est plus souvent question de la valeur émotionnelle des choses que de leur valeur numérique, informative et pécuniaire.

L'époque n'est pas uniquement matérialiste, elle cherche à rapprocher, fusse par quelques artifices superficiels et décoratifs, les différences qui fascine les hommes. Cette conquête n'est pas exclusivement dominatrice et coloniale. L'utopie occidentale et mondaine des Expositions Universelles, repose sur la classification des choses et caractérise cette folle accumulation. Abjecte, objecte, les figures sociales sont des armoiries corporatistes, les pays des bannières belliqueuses, les livres de lourds tomes picturaux, et ce fatras de signes, préambules d'une terrible défaite humaniste, la Grande Guerre, absurde et sordide bourbier qui engloutira ses belles espérances d'un savoir libérateur, préfigure des courants comme Dadaïsme et Surréalisme ou l'on jouera " comme en rêve " au grand mélange des images et des mots pour mieux s'en délivrer. Jules Verne est un homme de ce grand recensement, mais, transitant, c'est aussi un ambitieux rêveur. Bien avant que cette ère du savoir et de la raison ne paraisse si terrible et douteuse aux trahis et maudits de l'histoire, et qu'adviennent les plus grands et plus beaux amalgames conceptuels pour rire et pleurer des vanités et de la folie des hommes, ce monde inquiet recomptait inlassablement ses doigts comme un drôle d'enfant sage à l'aube des désillusions. Plus tard, le travail de montage et de déstructuration d'un rescapé des tranchées de la guerre de 14-18, le plasticien Max Ernst, présente en collage, une nouvelle surréaliste Une Semaine de bonté (1934). Il s'agit d'un très bel exemple de cette 
explosion de repères et d'images aliénés. Cet assemblage de gravures ressemble au mariage brouillé de planches pédagogiques et d'illustrations littéraires.

On croirait une sorte d'encyclopédie sans dessus dessous, un recueil d'archives secouées à la manière d'une boule à neige. On entrevoit le choc et le dépit dans ce rejet d'une époque maniaque de la conservation fragmentaire. En accumulateur d'images, d'effets et de pensées, on imagine volontiers, dans l'espace muséal de sa propre vie, un lieux de pèlerinage quotidien, le trésor intime de Verne l'entourant dans son cocon créatif. Objets, outils, instruments, modèles, répliques, le riche coffre de toutes ses passions, reliquaires, abécédaires, bestiaires, étagères de livres poussiéreux, de précieuses lunettes, celles qui regardent les astres ou celles qui épient le voisinage, une collection de cartes et de mappemondes, des compas de navigateur et de luxueux sextants.L'objet c'est le rêve ! On est loin des notions de valeur marchande, le bel objet n'est pas seulement rare, il est unique et de fait il ne s'achète pas toujours. Parfois il s'offre, se gagne, ou bien encore se trouve et se perd comme un secret, comme un trésor de pirate.

\section{Adepte : Un monde, des machines (Jules Verne et ses transportations)}

7 Imagé par d'illustres illustrateurs, Jules Verne participe activement à la conception graphique de son œuvre. C'est à croire que son travail serait (à son initiative?) indéfiniment en partage et toujours à compléter. Dessinateurs, musiciens, cinéastes, auteurs de Bandes Dessinées ou de spectacle vivant, tous apportent, dans une même révélation infantile, les pierres pluridisciplinaires d'un édifice savamment inachevé. Peut être est-ce là une belle représentation du mécanisme, qui ne sait bien longtemps garder son secrets et n'a de cesse d'être disputée ou partagée par les bricoleurs. Le travail inspiré de François Delarozière, créateur prodigue de nombreux accessoires de spectacle pour la compagnie Royal de Luxe, fait justement participer une machinerie rustique dans l'univers de la représentation. L'invention se distingue par sa fonction objective mais surtout par son fonctionnement apparent. Encore une belle citation et un attentif recyclage des rouages dramatiques de Verne à la sauce du théâtre à trappe : le voyage et la machine sont au cœur même des spectacles. Les célèbres et tous premiers "Géants " marionnettes-machines dont l'inspiration plastique certes très proche du personnage de Gulliver, rappelle aussi l'histoire de tous les véhicules et engins libres du voyage, de la conquête Vernienne du monde et sa vision onirique voir utopiste du progrès. Les spectacles Retour d'Afrique (1999) puis Les chasseurs de Girafes (2000) mettent en scène des marionnettes-automates géantes, une vraie-fausse machinerie dont toute les pièces ne servent pas techniquement, mues d'un désir très esthétique de mécanique ou d'aventure, parcouru de petits contes, le périple est aussi une syntaxe issue du bricolage.

L'inspiration fourre-tout de ces spectacles n'est pas un unique relevé topographique du seul monde Vernien et convoque aussi d'autres identités imaginaires. Le titre de leur création et hommage, conçu dans le cadre du centenaire de la disparition de Jules Verne : «La visite du sultan des Indes sur son éléphant à voyager dans le temps» (Royal de Luxe, Nantes, Mai 2005) ressemble à lui seul à un cadavre exquis des célèbres ouvrages de Verne et de Wells. La compagnie digère ses faisceaux créatifs comme un seul et même élan, une famille bien à part d'artistes machinistes. Autour des différents travaux entre souvent dans la partie, au coté de Jules Verne, Jonathan Swift pour le gigantisme (Les voyages de 
Gulliver - 1726) ou encore Herbert Georges Wells pour le voyage temporel (La machine à explorer le Temps - 1895). Ces appareillages d'apparence très bricolée restent le cœur d'un riche travail émotionnel. Il s'agit souvent de petits bijoux de technicité camouflés sous l'apparence des machines les plus rudimentaires et les plus saugrenues. Ces inventions, amies où ennemies, qu'elles fassent rire, peur ou rêver demeurent de grandes intuitions esthétiques. Nous vivons à la fois l'ère de la domotique et de l'intégration, l'invasion "sournoise et discrète" des microprocesseurs et en même temps sous l'emprise esthétique emblématique du style néo-rétro, de nos voitures-fusées au mobilier de grande série inspiré par l'Art Déco. Il faut voir et montrer la machine car autant que la force motrice du voyage elle est aussi le vecteur d'un discours. La machine incarne notre civilisation technologique, l'ère d'un modernisme victorieux ou inversement les peurs liées au progrès technique et à l'aliénation des individus (Les temps modernes - Charles Chaplin 1936) ou la remise en cause du vivant, du pensant et de la notions d'humanité (Do Androids dream of electric sheep? - Philip K. Dick 1968). Dans un tout autre registre stylistique, les terribles stratagèmes et autres bastions machiavéliques du Dr No, l'ennemi juré de James Bond (Ian Fleming) sont autant le spectacle que les fameux gadgets du héros. Que la machine, un véhicule spécial, un aménagement secret et insolite (un volcan...) ou une arme redoutable, soit bonne ou mauvaise, le plaisir intellectuel est bien moins manichéen que les caricatures hollywoodiennes.

La représentation esthétique de l'invention transcende parfois sa propre vocation, elle porte plus loin cette idée de l'Aventure et aiguise notre appétit d'apprentis sorciers. Ce rêve secret, l'île mystérieuse (1875), un Eden scientifique du capitaine Némo, ce père des " fous démiurges en technologie », est une sorte de machine suprême dont la fonction est celle de tous les instants : un idéal. Petit à petit le corps est conceptuellement devenu un organisme et le monde un écosystème. La notion de système et d'espace organisés et de mécanicité des fonctions motrices prend une place prépondérante dans l'imaginaire de ce dernier acte de la révolution industrielle. La transformation métaphysique du savoir laisse exploser, les peurs passées, les ambitions fantasques d'une science quasiprovidentielle. Savant fou, maître d'une science folle... où commence et s'arrête cette irascible envie de découverte et cette soif de connaissance? Verne invente, dit-on, le roman de géographie fiction, mais si son univers s'incarne dans quelques horizons nouveaux, il est animé d'une curiosité scientifique et soumis parfois à ses machines capricieuses. Les erreurs, les pannes et les avaries sont les signes prometteurs d'un idéal qui sort enfin du mensonge et de la tragédie chronique de la perfection. On peut y entrevoir la profanation d'un mysticisme religieux trop bien organisé. La machine est à l'image du et des créateurs, fragile et magique. Aussi incroyables, obscurs et abracadabrants que peuvent être leurs inventeurs, ces appareils prolongent, transportent, parfois perdent, bien au delà des seules limites, géographique. Voyager, c'est alors changer le monde et lui faire franchir les barrières conceptuelles et morales d'un contexte qui se radicalisent. L'ailleurs Baudelairien, une incitation addictive à la rêverie et au désordre fantasque, l'extraordinaire voyage Vernien d'autre part, un regard curieux et appliqué, humain et poétique, sur l'urgence d'une époque qui s'ouvre au monde devant tous les chaos, le départ étant aussi bien synonyme de découverte que de fuite. 


\section{Adapte : Une vision, un cinéma (Jules Verne et ses transpositions)}

10 Dans un no man's land émotionnel, une rare instance se tisse suspendue dans le petit laboratoire scolaire, quelques part dans les effluves sulfurées de la découverte et l'odeur âcre de l'encre sèche, un songe flottant entre la chaire professorale et la place du cancre rêveur. Verne crée des mondes, et ses mondes, parce qu'ils sont relativement plausibles, conservent souvent autant de repères et de phénomènes physiques qu'ils ne transgressent ouvertement les lois qui les régissent. Le caractère fondamentalement humain des passions et des velléités qui animent les personnages rendent généralement les mythologies accessibles voir progressivement crédibles. Le défit cinématographique n'est pas seulement de rendre convainquant l'univers Vernien, son invraisemblance faisant partie de ce que l'on appelle généralement un charme désuet, mais surtout de ne pas trahir la volonté d'un détracteur de cette forme de Science-fiction: faire rêver (voyager) scientifiquement. Il s'agit alors d'assortir son interprétation des phénomènes scientifiques, un parcours poétique, avec des d'effets spéciaux plus modernes et novateurs. Cinématographiquement (visuellement), comme pour la plupart des contes, ces mondes sont autant des espaces que des fonctions.

Le voyage est une transition mais aussi une transformation. La narration s'enroule, charmeuse, dans les méandres complexes d'un jeu de logique fou (flou ?) qui en rendrait la description plastique impossible si l'artiste ne tombait pas, à son tour, fort heureusement, entre les mains d'autres interprètes praticiens. Verne est un inspirateur inspiré. Il est, plus que dans la tradition cinéphile, constitué et restitué par ses pairs. La diversité de ses rencontres sont autant le reflet d'une époque, la saveur culturelle de la bohème, que la curiosité de l'homme. Ses tous premiers essais théâtraux cherchent très vite une forme de matérialité dans la musique. Son ami et compatriote Nantais Aristide Hignard, un malchanceux de l'histoire, s'y attelle à ses débuts ; l'expérience ne séduit pas vraiment et le duo ne trouve pas dans cette association texte et musique ce renouveau local de l'opéra qu'engendrerons plus tard les bouffes Parisiens. En effet, quelques années après, le plus célèbre et controversé compositeur d'opérette (et pas de pacotille), Jacques Offenbach accroche les idées de Verne, parfois contre son gré, à la portée des orchestres. Très vite et dans un genre bien particulier, ses histoires sont présentées et incarnées par des acteurs. On cite souvent, «d'après une nouvelle de Jules Verne », ces opéras bouffes tel que Le Docteur Ox (recueil de nouvelles paru en 1874 et adapté en 1877) ou encore Voyage dans la Lune (1875) une farce très inspirée (et très irritante!) de De la terre à la Lune (1872) et Autour de la Lune (1870).Verne est porté à l'écran par ses contemporains dès 1901. Charles Pathé s'essaie sur Les enfants du capitaine Grant puis, l'année suivante, Georges Méliès introduit avec Le voyage dans la Lune une imagerie extraterrestre devenue culte. Ce dernier réalisera les premières transpositions cinématographiques des romans. Verne profite, in extremis, d'être au cœur des tous premiers films de l'histoire et devient, comme par un signe du destin, l'un des premiers auteurs vivant adapté au cinéma ! Qu'il le veuille ou non - parfois les projets lui déplaise - en faisant rêver certains des plus glorieux de ses contemporains, c'est toute une époque que Verne emmène sur la Lune.Dans la pratique audiovisuelle les espaces et les fonctions s'appellent des décors et effets (esthétique et technique). 
Réaliser Jules Verne c'est avant tout confectionner et élaborer des lieux et des appareils fantastiques. La réalisation filmique, jeu d'illusions bien plus dispendieux que la littérature, exauce parfois les formules mais rarement toute la magie. Le mythe re-visité opère une double implication stylistique. Il s'agit à la fois de recréer l'instrument, le dispositif ou l'appareil d'origine, celui qui coïncide historiquement avec l'époque de Verne et des protagonistes de ses romans du point de vue esthétique et technique et, en même temps, de moderniser, d'harmoniser le tout pour que les effets spéciaux et les créations plastiques gardent une efficacité contemporaine. Cette efficacité stylistique n'est, par ailleurs, pas toujours le signe d'une démission conceptuelle comme nous inflige actuellement nombre de super-productions Hollywoodiennes. Je ne parle pas d'un corpus de nécessité c'est à dire de retrouver "les bonnes ficelles qui marchent », le prorata de Hi-Tech et la star en vogue, mais plutôt d'offrir au spectateur une émotion équivalente, une force imaginative à la hauteur (amplitude inestimable) du roman lors de sa sortie. Jules Verne est un homme d'effet, il connaît la portée puissante de l'émotion dans le discours, il œuvre à la fois en auteur et pédagogue dans l'espace d'une double mission que lui confie Hetzel son éditeur : cultiver et distraire.

Ce pur concept idéologique de la pensée laïque, régulièrement controversé et remis en cause dans son efficacité, sur lequel se base encore, fort heureusement, l'engagement de cette chère vieille dame diffère très nettement du rendement imposé par l'industrie cinématographique. Verne est un "honnête » marchand de sensation(nel), dans une certaine idée de la noblesse intellectuelle, à l'instar de Georges Méliès (Le voyage dans la Lune - 1902), il livre le travail d'une recherche aussi étonnamment laborieuse que ludique, avec le sérieux de grands enfants, culturellement dépositaire et non-plagiaire, fruit d'une véritable science de la confection. L'esthétique résiduelle, ce que l'on appelle communément "l'univers Jules Verne» est tout aussi fondamentale que son style littéraire qui reste, d'après lui même, assez anonyme. Pour Harper Goff, qui créera le design du Nautilus dans l'adaptation oscarisée de 20000 lieues sous les mer par l'équipe Disney (1954), il s'agit d'imaginer une fusion esthétique et technique qui rende compte au mieux de deux époques du fantastique : L'univers de Verne / Némo et son style Victorien (ou second Empire en France) post-industriel et en même temps le design d'après guerre, célèbre époque d'une science-fiction très «électroménager ». Le fantasme technologique de l'Amérique des années 50 est résolument tourné vers la conquête spatiale et le mythe entier du pionnier remanié à la sauce martienne. Cette adaptation sort entre The day the Earth stood still (1951) et Forbiden Planet (1956) cultissimes films de SF fait de robots humanoïde d'arme et de vaisseaux spatiaux dans le pur style "grille-pain ». La science n'est qu'un frêle esquif, parfois amusant parfois triste, qui traverse, toujours à la limite d'une rupture, l'univers le plus étrange qui soit : le nôtre. L'œuvre de Verne est à la fois faite de "belle mécanique » et est toute entière un grand dispositif esthétique. Il s'agit, plus que de roues réinventées, d'une gigantesque révolution. Anachronisme délicieux, qui colle à son auteur et honore sa réelle inventivité : la machine Jules Verne est fondamentalement audiovisuelle.

Si Verne n'est pas l'inventeur du sous-marin il est sûrement l'un des initiateurs de la vision sous-marine, entraînant dans la fascination un siècle de désir technologique. Le Commandant Cousteau, rêvant d'un œil cinétique de grands fonds comme de faune et de flore côtière, est le plus inspiré exemple de personnage audiovisuel emprunté au Capitaine Nemo. L'idole est certes bien plus littéraire et libertaire que le documentariste moralisateur et elle invente ce nouveau genre d'aventurier en scaphandre, assez aimant 
pour chercher sans fin et assez fou pour ne rien voir... notamment du danger. Si c'est aux sciences «bien à l'armée » que l'on doit les sous-marins, on les imagine volontiers « à la Jules Verne", c'est à dire avec de larges hublots vitrés, une coûteuse lubie sous l'incroyable pression de 20000 lieues de fond, dans l'espoir de voir, dans cette obscurité absolue, ce que les sonars actuels n'arrivent même plus à identifier. Verne n'invente pas, il s'approprie. Et cette belle outrecuidance, novatrice dans les domaines technologiques, inaugure une liberté esthétique qui décomplexe entre autre la création audiovisuelle jusqu'à son paroxysme actuel: ne plus attendre des sciences qu'elles nous montrent quelques "vérités bien réelles ", à l'instar de ce que l'on attendait des religions, mais exiger d'elles qu'elles réalisent véritablement nos désirs. Verne inspire formellement le cinéma par son inventivité et son style mais c'est aussi sa « méthode » tout entière qui est happée par le dispositif. Du principe scientifique et technique que détourne les saltimbanques (théâtre, photo, cinématographe, etc.), en quête de sensations nouvelles pour leur spectacle, on arrive au spectacle résolument scientifique. Faire le tour du monde en 80 jours tiens de la même démesure et arrogance que de " faire 80 mondes le tour d'un jour ». So british, Phileas Fogg est un peu de la trempe de ses reporters pressés qui parcourent sans plaisir les paradis terrestres dans l'urgence de la performance et de l'efficacité. C'est un gentleman et sa haute condition transforme le voyage en un tourisme aveugle, pécunier et pragmatique. Le journalisme moderne n'as pas vraiment l'air plus attaché aux lieux et aux peuples que ce nanti héros. Verne imagine le temps d'un défit à l'Anglaise l'illusion de notre journal télévisés, le tour de la planète chronométré, la quantité et la vitesse.

Cette palette picturale des zones occidentalisées, cette débauche de moyen pour couvrir les quatre coins du globe rappelle la frénésie caricaturale du 20 heures. Après tout l'ORTF ne s'était elle pas donné, vis à vis du téléspectateur, des buts assez similaires que le magasin d'éducation d'Hetzel? L'ambition n'as t-elle pas succombé à la tentation de la performance marchande? Seule la télévision et le cinéma sont actuellement capable de rentabiliser une telle couverture et un tel envahissement systématique du monde. Là encore la pauvre inspiration des concepteurs pioche indifféremment dans toute la littérature de science fiction du XIX ${ }^{e}$ siècle. Mis dans la peau de Robinsons, les joueurs de certains reality shows sont artificiellement isolés sur des îles désertes reconstituées pour s'approcher du roman de Daniel Defoe. Les émissions du petit écran empruntent beaucoup au mythe d'aventuriers «à la Jules Verne». Nicolas Hulot, présentateur " aventurier » est un adepte inconditionnel des montgolfières et autres ailes volantes. La fascination pour ce bric à brac roulant, flottant ou volant tient bien moins de la pure performance technique que du spectacle visuel en soit. L'audiovisuel a trouvé dans les machines Verniennes l'emblème iconographique de l'aventure et un projet lointain de vulgarisation scientifique. 


\section{AUTEUR}

\section{FRÉDÉRIC CASSAN}

Doctorant au Lara. ATER Lara / Esav. Université de Toulouse II 